\title{
Teaching pharmacy law: How to engage students in the context of large group lectures
}

Author: Ellen I Schafheutle. Manchester Pharmacy School, The University of Manchester

Background: The teaching of legal requirements underpinning pharmacy practice is a core requirement in MPharm degrees. Learning of medicines related activities is commonly applied in 'practice of pharmacy' practicals, with theoretical background taught in large group lectures. Lectures are a widely used and efficient form of delivery, but they are passive and student attention can wane. The redesign of law lectures aimed to increase interactivity and student engagement; evaluation aimed to assess student acceptability and satisfaction.

Method: TurningPoint ${ }^{\circledR}$ polling software ('clicker' questions) were integrated throughout every law lecture. Students were encouraged to use 'Medicines, Ethics and Practice' (MEP) by leaving large blanks of core requirements in student handouts. Following key topics, such as prescription requirements, controlled drugs and fitness to practise (ftp), a lecture was dedicated to support application through interactive revision questions, prescription examples or ftp cases. Online tests with automated written feedback supported student revision and exam preparation. TurningPoint ${ }^{\circledR}$ was used for the end-of-semester evaluation of two year 2 and one year 3 cohort of about 160 students between 2011/122012/13.

Results: Per cohort 80-93 students responded. Almost all (93\%-98\%) appreciated the importance of a good understanding of law. With increasing interactivity incorporated into lectures over time, the proportion of students agreeing they enjoyed law lectures increased from $42 \%-56 \%-74 \%$. Thirty-nine to $46 \%$ appreciated the importance of the MEP and accepted gaps in handouts, $22-30 \%$ being neutral. Most (90-98\%) found interactive prescription examples useful, and $76-92 \%$ thought an interactive revision lecture was valuable. About a third of students accessed the formative tests during term-time; the majority completed these nearer the final exam. Of those who accessed the tests, most $(86 \%-96 \%)$ found the instant written feedback valuable.

Conclusion: 'Clicker' questions during law lectures, supported by online formative resources, increase interactivity and support student learning, making a relatively dry subject engaging and enjoyable. 